\title{
The phase equilibrium phenomenon in model hydrogenation of oleic acid
}

\author{
Ana R. C. Morais • Andre M. da Costa Lopes • \\ Rafal Bogel-Lukasik
}

Received: 2 March 2014/ Accepted: 20 June 2014/Published online: 23 July 2014

(C) Springer-Verlag Wien 2014

\begin{abstract}
Hydrogenation is one of the most commonly practised types of reaction in industry. The processing of low or null price feedstock to produce energy through hydrogenation is an interesting solution for waste valorisation. The hydrogenation in $\mathrm{CO}_{2}$ atmosphere offers a series of advantages and facilitates the process by the dramatic reduction of normally harsh reaction conditions. The hydrogenation of natural feedstock with complex matrix is a challenging task and the examination of the phase equilibrium of this system is crucial to understand the phenomena driving the kinetics of the hydrogenation reaction. High pressure phase equilibrium modelling is a key method to design and to analyse the obtained data and helps to understand the hydrogenation reaction outcome. The increase of $\mathrm{H}_{2}$ pressure does not translate to the increase of hydrogen solubility in the liquid phase due to the significant decrease of $\mathrm{CO}_{2}$ solubility in oleic acid. The obtained data confirm that both thermodynamics and kinetics play an important role in the hydrogenation of cattle fat in the presence of $\mathrm{CO}_{2}$.
\end{abstract}

Keywords Hydrogenations .

Supercritical carbon dioxide $\cdot$ Fatty acids $\cdot$ Hydrocarbons . Phase diagrams

\section{Introduction}

The renewable resources start to play an important role in the satisfying the continously increasing demands for

A. R. C. Morais · A. M. da Costa Lopes · R. Bogel-Lukasik ( $\square)$ Unidade de Bioenergia, Laboratório Nacional de Energia e Geologia, I.P., Estrada do Paço do Lumiar 22, 1649-038 Lisbon, Portugal

e-mail: rafal.lukasik@lneg.pt energy supply contributing to the sustainable development. The obligation established by the renewable energy directive created by European Commission in [1] endorsed a mandatory share of a $10 \%$ for biofuels and other renewable fuels in transport sector consumption by 2020 to be achieved by all EU Member States. One of the methods to reach this target and to avoid food versus fuel dilemma is the use of second and third generation feedstock. Additionally, these types of feedstock or especially the use of wastes and residues is favoured, as this offers a much more competitive price of the final biodiesel and opens a new route to recycle and to valorise these raw materials. The major hindrance in the valorisation of this resource is a need of new and more advanced technologies comparing with currently existing ones for the first generation biofuels. Wastes and residues rich in free fatty acids are much easier to be transformed and one of the potential processes to do this is hydrodeoxygenation. The product of hydrodeoxygenation is $\mathrm{H}$-oil and is formed in the catalytic hydrogenation and cracking process. The principal aim of this conversion route is the removal of oxygen and simultaneous hydrogenation guiding to formation of hydrocarbons. Depending on the process conditions, the produced fraction contains hydrocarbons similar to gasoline or to diesel $[2,3]$. Additionally, the produced H-oil possesses the characteristics superior to classical fossil fuel and the downstream processing is much simpler than in case of classical esterification. The major problem of the hydrodeoxygenation is the need of high solubility of $\mathrm{H}_{2}$ in the liquid phase and adequate catalyst activity that is strongly dependent on temperature. The solubility of gases in liquids is very limited therefore the use of high pressure of $\mathrm{H}_{2}$, even up to 100 atmospheres, is required [4]. Additionally, the noble metal catalysts generally require high temperature (even $673.15 \mathrm{~K}$ ) [4], guiding in parallel to 